\title{
Collaborations syntaxiques - Formes et fonctions de leur usage dans un groupe subculturel lyonnais
}

\section{Frank Ernst Müller et Sabine Klaeger}

\section{(2) OpenEdition}

\section{Journals}

\section{Édition électronique}

URL : http://journals.openedition.org/pratiques/1666

DOI : 10.4000/pratiques. 1666

ISSN : 2425-2042

\section{Éditeur}

Centre de recherche sur les médiations (CREM)

\section{Édition imprimée}

Date de publication : 15 décembre 2010

Pagination : 223-243

Référence électronique

Frank Ernst Müller et Sabine Klaeger, «Collaborations syntaxiques - Formes et fonctions de leur usage dans un groupe subculturel lyonnais », Pratiques [En ligne], 147-148 | 2010, mis en ligne le 15 décembre 2010, consulté le 20 avril 2019. URL : http://journals.openedition.org/pratiques/1666 ; DOI : 10.4000/pratiques. 1666 


\title{
Collaborations syntaxiques - Formes et fonctions de leur usage dans un groupe subculturel lyonnais
}

\author{
Frank Ernst Müller \\ Universität Mannheim und Frankfurt/M., Allemagne \\ Sabine Klaeger
}

Universität Bayreuth, Allemagne

\section{Données et propos de notre étude}

Notre étude porte sur des formes de collaboration syntaxique (désormais : CS) dans les interactions quotidiennes, un phénomène qui a suscité l'intérêt de l'Analyse Conversationnelle (désormais : AC) dès ses débuts. Ainsi, Harvey Sacks, un des fondateurs de l'AC, a souvent cité et discuté l'exemple suivant d'une telle collaboration, exemple pris d'une conversation parmi des jeunes américains de même âge :

(1) Ex. Sacks (1995:321)

Ken: $\quad$ We were in an automobile discussion,

Roger : discussing the psychological motives for

( ): hhhhhhh

Al: $\quad$ drag racing on the street.

Les trois locuteurs produisent, sans césure ni coupure, pour ainsi dire sans point et virgule (dans la transcription de Sacks, la virgule se rapporte uniquement à l'intonation montante) un seul énoncé cohérent qui englobe et intègre les trois participants. L'on peut résumer l'interprétation de Sacks de la manière suivante : en s'articulant de cette manière collaborative, à travers d'une «coénonciation » (Jeanneret 2001), les trois jeunes gens, par leur façon de parler, peuvent «prouver », c.à.d. démontrer interactivement, qu'ils constituent un groupe d'amis, une équipe bien rodée dans la matière en question.

Depuis les premières observations de Sacks dans les années soixante, beau- 
coup d'études ont été consacrées à ce sujet ${ }^{(1)}$. On a démontré, par exemple, que les CS existent dans une grande variété dans leur réalisation syntaxique, prosodique et interactive, qu'elles touchent la structure conversationnelle en général et qu'elles ne se limitent pas à des contenus ou à des locuteurs spécifiques. Malgré ceci, notre propos dans le cas présent ici reste près de l'orientation esquissée par Sacks : nous allons nous intéresser au rôle et aux fonctions que peuvent avoir ces formes assez marquées de collaboration à l'intérieur des pratiques communicatives d'un groupe donné, groupe au sujet duquel nous disposons d'un corpus considérable et dont nous avons étudié les interactions verbales au quotidien. Nous n'avons donc pas cherché à rassembler une collection d'exemples pertinents venants de corpus variés, comme c'est le cas dans presque tous les travaux sur ce sujet. Tout au contraire, nous avons changé la hiérarchie des priorités : excepté les exemples qui servent dans la première partie à introduire la découverte des phénomènes syntaxiques et conversationnels par l' AC, c'est uniquement à l'intérieur d'une seule recherche qui place au centre de son intérêt l'observation et la description du style social d'un groupe donné que nous allons aborder notre objet, à savoir les CS et les formes de coénonciation qui en résultent. Il s'agit d'un groupe de squatteurs de Lyon qui a été décrit dans un portrait sociostylistique par Klaeger $(2007)^{(2)}$. En regardant nos exemples, nous essayons d'analyser la valeur des CS, leurs fonctions dans l'usage interactif de ce groupe, notamment dans leurs réunions et débats qui sont caractérisés par la familiarité des rapports mutuels entre ses membres vivant en communauté.

Parmi les hypothèses qui essayent d'établir un rapport entre l'aspect formel des structures conversationnelles d'une part et l'aspect communicatif et ethnographique d'un groupe social d'autre part, citons ici celle de Pelose (1987) et celles de Coates (1994). Selon Pelose (1987), ce sont surtout la bonne connaissance de la matière discutée ( «topic familiarity ») et le caractère informel et intime des relations à l'intérieur d'un groupe («intimacy of relationships ») qui jouent un rôle important et favorisent l'emploi des CS. Coates (1994) analyse des «turn-taking patterns in the talk of women friends ». Elle affirme que l'on peut trouver une stratégie marquée dans les pratiques des prises de parole, stratégie qu'elle appelle « partage de l'espace locutoire» («floor-sharing »). Dans le floor-sharing, un principe fondamental de l'organisation des tours de parole, le principe de « un locuteur à la fois », tel qu'il a été décrit par Sacks/Schegloff/Jefferson (1974, désormais : Sacks et al.) est temporairement suspendu : «Through the construction of a shared floor, the group as a whole rather than any individual speaker arrives at a summary of the topic they have been discussing 》 (Coates 1994 : 182). Les notions développées dans ces études nous serviront en tant que grille d'attention pour focaliser les observations auprès de nos propres données.

(1) Nous ne citons ici que quelques études parmi les nombreux travaux sur ce thème : Coates (1994), Gülich (1986), Jakoby/Ochs (1995), Jeanneret (1999, 2001), Kallmeyer (1999), Lerner (1991, 1993, 1995, 1996), Mondada (2007), Müller (1995), Sacks (1995), Schmale (2008).

(2) Les squatteurs vivent à la La Lutine, un immeuble dans le $7^{\mathrm{e}}$ arrondissement de Lyon. L'analyse qualitative de Klaeger (2007) montre comment, dans ce milieu alternatif urbain, une identité sociale spécifique est construite à travers un style de communication, et dans quelle mesure les valeurs sociopolitiques qui y sont revendiquées influent sur les normes communicatives du groupe. 
Dans la suite, il s'agira de décrire d'une part les propriétés formelles et structurelles de la CS, et d'autre part de situer ces structures dans une description des pratiques communicatives du groupe.

\section{Principes de base}

\subsection{Le rôle de la syntaxe dans l'AC}

L'intérêt que l'AC a développé dans le champ de la syntaxe ne s'oriente pas vers la grammaire « traditionnelle » avec ses règles abstraites; c'est au contraire celui d'une «syntax-for-conversation »(Schegloff 1979). L'intérêt se porte donc sur les fonctions qu'il faudra accorder à la syntaxe et à l'intonation en tant que moyens qui servent à la construction des unités primaires découvertes et spécifiées par la théorie de l'AC, c.à.d. les tours de parole et l'alternance des tours de parole dans la conversation («turn-taking »). Une observation élémentaire de Sacks et al. (1974) était le fait que les participants d'une conversation arrivent à prendre la parole et à la céder d'une manière ordonnée, qu'ils s'enchaînent en minimisant aussi bien les silences que les chevauchements et qu'ils agissent de façon à ce qu'il n'y ait qu' " un locuteur à la fois ». La question ouverte par ses constats apparemment très simples consiste à se demander « comment les locuteurs effectuent cette alternance d'un tour à l'autre avec précision, systématicité et méthodicité, sans qu'ils aient à se concerter d'avance ou à en thématiser explicitement le moment et la manière » (Mondada 2007 : 7). Un aspect important dans la gestion de l'alternance des tours pour les interlocuteurs est d'identifier le moment précis de l'alternance. Celle-ci se règle «localement » dans l'interaction : chaque fois qu'un participant atteint, dans sa construction d'un tour (« turn constructional unit », TCU) un point de complétude (possible), la question de qui prendra la parole se pose de nouveau. Ce sont donc les moments où les tours de parole arrivent à une complétude (possible), qui sont « pertinents pour la transition » («transitional relevance points », TRPs). Il est évident qu'un participant qui est récepteur silencieux à un moment doit suivre, en détail et avec attention, l'émergence et le déroulement d'un tour pour identifier le moment précis de l'alternance et pour devenir locuteur à un autre moment ${ }^{(3)}$. Dans un modèle de ce genre, il est évident aussi que l'aspect temporel, l'émergence des TCUs dans le temps et l'attention synchronisée des participants envers cette émergence auront une grande importance.

Pour construire un tour, la syntaxe n'est pas le seul moyen; l'unité qui constitue un tour de parole peut par exemple consister en une seule syllabe ou en un seul geste. Mais les constructions plus complexes en forme de syntagmes et de phrases sont les TCUs les plus intéressantes : l'articulation lexicale, syntaxique et prosodique permettent, dans la trajectoire de leur émergence, une projection et une prédiction quant à leur continuation et à leur achèvement :

(3) Ainsi, par l'orientation permanente pour l'alternance, qui est intégrée dans la conversation - du moins dans la conversation «naturelle », « autogérée par les participants » (« self-administered by members »), la conversation crée une « motivation intrinsèque » (Sacks et al. 1974 : 727) pour l'écoute attentive. 
sentential constructions are capable of being analysed in the course of their production by a party/hearer able to use such analyses to project their possible direction and completion loci. In the course of its construction, any sentential unit will rapidly (in conversation) reveal projectable directions and conclusions, which its further course can modify, but will further define. (Sacks et al. 1974 : 709)

Les CS soulèvent un nombre de questions. Ceci concerne surtout la notion centrale de l'AC, celle du tour de parole, les confins et l'intégrité des tours de parole, leur "perméabilité » (Lerner 1996) et surtout les pratiques et les modalités des participants qui s'insèrent dans un tour en cours, qui « appartient » déjà à un autre locuteur : dans les CS, les participants prennent la parole à l'intérieur du tour d'un autre («within-turn-talk») ${ }^{(4)}$. S'insérer dans le tour d'un autre équivaut en général à s'insérer dans une construction syntaxique en train de se faire.

\subsection{Temporalité, synchronisation des participants, « precision timing »}

Une découverte, faite déjà assez tôt dans l'évolution de l'AC ( $c f$. notamment Jefferson 1973) est le rôle important qu'il faut accorder à la dimension de la temporalité. Dans l'observation minutieuse de données enregistrées et transcrites, l'interaction se révèle être une « œuvre de précision » où les actes des participants sont ajustés les uns aux autres et coordonnés dans leur émergence en temps réel. Parmi une grande variété d'observations sur l'organisation temporelle, les CS et leur coordination en temps réel étaient dès le début un objet de grand intérêt. Dans ces constructions se montre la « capacité technique » (Jefferson 1973) avec laquelle un participant (récepteur et prochain interlocuteur) peut prendre la parole et placer sa contribution dans une structure - pas encore terminée et toujours en émergence - initiée par l'interlocuteur précédant avec une telle précision que la «continuation » est intégrée sans aucun retard perceptible ${ }^{(5)}$. Les participants sont synchronisés dans la perception mutuelle de leur interaction présente et s'orientent ensemble online, sur une même ligne de progression temporelle de leurs actions. Les constructions syntaxiques en cours de progression peuvent donc être analysées instantanément par les participants et doivent également être prises en considération dans leur émergence et leur déploiement temporel par l'analyste, à l'intérieur d'une «online-syntax » (Auer 2007).

Un ensemble de phénomènes observables qui témoigne d'une telle analyse online des trajectoires syntaxiques en émergence a été décrit dans une série d'études de Jefferson (1973, 1984, 1986). Il s'agit de plusieurs types de (co)énoncés qui sont placés en chevauchement («overlap »). Tandis que les che-

(4) Les recherches sur les CS reflètent souvent déjà dans leur titre et leur terminologie ce statut du « within-turn-talk». Cf. par exemple Kallmeyer (1999), qui appelle les phénomènes en question " others' inserts », ou Lerner (1996) qui parle de « conditional entry into the turn space of another speaker».

(5) Selon Jefferson, le récepteur d'un énoncé en cours a donc la capacité « to select a precise spot to start his own talk "no later" than the exact appropriate moment to place it so that it will sound like a "continuation" of the prior/ongoing talk. [...] That is, the ongoing speaker's "continuation" and the recipient's ,continuation' can be seen as simultaneously started phrases or clauses, with absolutely no gap between the former and latter » (Jefferson $1973: 51-52)$. 
vauchements sont en général évités ou traités comme faits accidentels, résultants d'un manque de coordination parmi les participants, ils sont, dans le cas présent ici, tout au contraire vus comme le résultat d'une performance verbale hautement organisée et même élaborée : ils s'adressent à un objet particulier dans le tour émergent et, dans leur placement exact, ils «visent » cet objet. De tels chevauchements, qui représentent des exemples d'un " achieved overlap », (Jefferson 1984), i.e. d'un " chevauchement accompli » réalisé de manière ordonnée et coordonnée par les participants, se trouvent dans les exemples (2) à (4). Ces exemples proviennent d'un débat sur l'orthographe du français ( $c f$. Müller 1995, 1999).

(2) Ex. Müller $(1995: 33)^{(6)}$

01 W1: c'est d- là tu reconNAIS que l'orthoGRAphe euh de quelqu'UN [ révèle son éducatiON (7) $02 \Rightarrow \mathrm{W} 3$ :

[ ça révèle

L'exemple (2) présente une coénonciatrice qui insère sa contribution en parfaite simultanéité avec la même unité lexicale réalisée à ce moment dans la structure émergente par le locuteur en cours. On peut même supposer qu'elle emploie la forme brève du pronom - ça, en 02 - pour pouvoir accomplir le chevauchement d'une manière exacte ${ }^{(8)}$. Elle arrive ainsi à dire la même chose au même moment que l'interlocuteur en cours. Dans un tel cas, le chevauchement peut documenter l'unanimité parfaite avec l'interlocuteur en cours - une co-orientation tellement effective qu'elle permet d'anticiper exactement ses paroles à ce moment-là dans la structure émergente développée jusqu'à ce point et de les articuler en même temps. Jefferson (1984) parle dans ces cas d'un « savoir indépendant» (« independent knowledge»), puisque le coénonciateur réclame, avec son parallélisme manifesté, d'être arrivé ,indépendamment' au même résultat.

S'il est vrai que la coénonciatrice en (2) s'insère dans le tour d'un autre, sa participation est évidemment de nature supportrice : «timing » et placement de l'énoncé coproduit, ainsi que sa brièveté, démontrent que son intervention est « taillée » de façon à s'accommoder au cadre syntaxique et sémantique de ce tour et ne met nullement en question le droit local de la locutrice en cours à le poursuivre $^{(9)}$.

Dans les « chevauchements accomplis », précision et synchronisation sont de rigueur : un léger décalage suffirait déjà pour en changer l'interprétation interactive et détruire le parallélisme qui supporte l'effet d'unanimité. Si l'unité coénoncée arrive trop tard, elle sera interprétée seulement comme une reprise d'un mot qui a été utilisé par le locuteur en cours et qui a été choisi par celui-ci; si, par contre, elle arrive trop tôt, elle sera forcément interprétée comme une formula-

(6) Pour les conventions de transcription, $c f$. 1'annexe.

(7) Pour la transcription et l'analyse de l'exemple dans toute sa longueur, $c f$. Müller (1995 : 334-338).

(8) Pour accomplir un placement précis de leurs (co-)énoncés dans le déroulement du tour en cours, les locuteurs emploient aussi d'autres formes de compression, par exemple la compression de syllabes à l'intérieur des mots polysyllabiques ou l'accélération momentanée du débit de parole, $c f$. Lerner (1996:246-247).

(9) Selon la description de Kallmeyer (1999), les «insertions » (« others' inserts ») possèdent les trois caractéristiques suivantes : a) subordination syntaxique; b) «next positioning » (placement auprès de l'objet qui est visé) ; c) brièveté. 
tion qui est proposée au locuteur en cours et la reprise par celui-ci comme une confirmation de cette proposition; si elle est insérée dans une « lacune » ( gap », cf. Sacks et al. 1974) dans la chaîne parlée du locuteur en cours, elle sera interprétée comme réparation et sa reprise par le locuteur en cours comme ratification de la réparation proposée. C'est ce dernier cas des lacunes quand la progression ultérieure d'un tour de parole (et d'une structure syntaxique) initié, mais manifestement encore inachevé, est troublée, qui provoque les achèvements collaboratifs les plus fréquents dans nos données.

Si l'on considère l'emplacement de l'élément coénoncé en (2) par rapport à sa place dans la structure syntaxique émergente, il se situe assez tard dans son développement. Egalement tard dans la trajectoire émergente se réalisent les coénonciations en (3), qui s'articulent dans un « chevauchement terminal» (« terminal overlap ») ou alors une « complémentation anticipée » (« anticipated completion », Jefferson 1984) : C'est seulement vers la fin d'une structure syntaxique et sémantique qui est déjà largement construite et à laquelle ne manquent que peu de constituants pour la compléter d'une façon minimale que les coénonciations apparaissent.

(3) Ex. Müller (1995: 336)

01 W1: quand je reçois une lettre de quelqu'un la première fois bon je remarque les fautes

02 \&d'orthographe (.) ca peut être mon meilleur ami ou ma meilleure amie je remarquerais

03 \&encore les fautes d'orthographe (.) mais j'y ferais pas- j'y ferais pas attentiON si tu veux

$04 \quad$ \&j'y attacherais pas d'imporTANce mais je [les remARquel

05 M2: [oui mais ça te gène dans la lecTUre et aussi

06 \&dans la compréhensiON hein/

07 W1: non non je n' peux pas dire que ça gène au niveau de la compréhension tu sais quand

08 \&quelqu'un t'écrit 'er' au lieu de 'e' accent aigu 's' -(.) [tu peux comprendre

$09 \Rightarrow \mathrm{M} 4$ : [ tu comprends

$10 \Rightarrow M: \quad$ [ tu comprends

Les coénonciateurs peuvent évidemment exploiter les ressources de la projection qui existent aussi bien sur le plan syntaxique que sur le plan sémantique et pragmatique d'un échange en cours. Comme on l'a vu supra ( $c f$. la citation de Sacks et al. 1974), les trajectoires syntaxiques admettent des projections séquentielles qui se spécifient et deviennent de plus en plus précises au cours de leur progression vers la fin d'une phrase ${ }^{(10)}$. Ainsi, dans (3), la construction de la phrase subordonnée, qui est introduite par quand(07), précède la coénonciation. Elle est ainsi l'élément premier d'une phrase complexe qui projette, après sa fin (marquée par la prosodie) l'arrivée d'un deuxième élément à suivre, qui est spécifié syntaxiquement en tant que phrase superordonnée. Et c'est précisément là,

(10) $C f$. les travaux de Auer $(1996,2002,2007)$ à propos de la projection syntaxique. En parlant de la prévisibilité séquentielle des structures émergentes, il note : « while participants go through a phase of maximal planning during the early parts of a trajectory, in which emerging structures have to be constructed and understood, the amount of processing effort decreases during its course : usually the final parts can be predicted» (Auer 2002:2). Dans cette perspective cognitive, la " collaborative sentence completion 》 (Sacks et al. 1974) prend l'aspect d'un véritable gestaltschluss. Dans la discussion de nos exemples, nous allons commenter chaque fois - en bref - cet aspect de la projection/de la prévisibilité syntaxiques. 
à la frontière syntaxique majeure de la période, que s'insèrent ici les deux coénonciations ${ }^{(11)}$

Le gestaltschluss et la co-construction dans ce format de phrase complexe ( $s i$ $p$-alors $q$ ) n'ont pas uniquement l'aspect de la complémentation syntaxique déjà mentionnée. Ils peuvent se rapporter également, ainsi que c'est évident en (3) et (4), à l'enchaînement argumentatif de deux propositions, en particulier à la conclusion $q$ à tirer de $p$, et à l'évidence de cette conclusion. La séquence argumentative en (3) intègre plusieurs participants et surtout les coénonciateurs M4 et $\mathrm{M}$, qui prennent position : ils soutiennent l'évidence et la validité de la conclusion tirée par W1 en la renforçant, par le savoir indépendant de leur chevauchements accomplis, dans son émergence même. Il s' " alignent » ainsi avec l'argumentation de $\mathrm{W} 1$ et se distancient en même temps de la position opposée de M2.

Le même aspect - renforcer l'évidence d'une conclusion à tirer in statu nascendi - est visible en (4). Dans l'essai de « dire la même chose au même moment », la coénonciatrice adopte même la perspective personnelle de la locutrice en cours :

(4) Ex. Müller (1995 : 72)

01 W1: j'fais comme tout le monde hein (.) quand je le- quand je sais pas comment

02 \&écrire un mot si j'ai un dictionnaire je chERche et si j'ai pas de dictionnaire

03 \&bon ben (.) [j'écris comme je CROIS

$04 \Rightarrow$ W4: [j'écris comme je peux

Les jonctions structurellement importantes sont souvent, comme ici, le « lieu » où sont localisées des interventions insérées dans la genèse d'un tour de parole/d'une construction syntaxique en progression. Souvent, les jonctions importantes sont également le lieu de césures marquées par l'intonation, par des hésitations, des micro-pauses, des allongements de voyelles ou d'autres procédés préparatifs de la part des interlocuteurs qui servent à organiser la formulation des composantes à suivre (sur cet aspect, $c f$. Schmale 2008). Ces césures sont grammaticalement prévisibles et constituent une partie structurelle de ce que Lerner (1996) appelle la «perméabilité » des tours de parole. Elles ouvrent, sur une micro-échelle de l'émergence de la chaîne parlée, chaque fois un " espace d'opportunité » permettant d'initier à ce moment une intervention, très souvent marquée par les trois traits déjà nommés : subordination syntaxique, next positioning et brièveté ( $c f$. Kallmeyer 1999) ${ }^{(12)}$.

Dans l'exemple suivant, la jonction où s'insère le coénonciateur est la frontière syntaxique et prosodique entre syntagme nominal et phrase relative. On reconnaîtra ici la présence du jeu de la projection séquentielle bipartite : la première composante contient un élément cataphorique dans la phrase nominale (tous les honneurs politiques et autres, 02/03), qui renvoie à une deuxième composante, à savoir la phrase relative attachée (qui, etc.) qui va la spécifier :

(11) $C f$. la discussion détaillée des périodes syntaxiques composées bi-partites en anglais du type de if $p$-then qu et when $p$-then qu et les exemples de leur co-construction fréquente dans Lerner (1996).

(12) Il est vrai que Kallmeyer (1999) trouve aussi des cas dans son corpus où une intervention qui s'annonce initialement comme une brève insertion pour compléter un tour, se développe dans la suite et finit comme un véritable tour de parole d'un « occupateur». 
(5) Ex. Mondada (1999: 25)

01 E: René Brouillet a refusé de signer notre satisfecitl et pour

02 \&moi c'est là bien la preuve qu'il méritait tous les honneurs

03 \&politiques et autres/ [qui dont il a bénéficié ensuitel

$04 \Rightarrow \mathrm{P}: \quad \quad[\mathrm{mhm}$ qu'il a eus plus tard

Le coénonciateur essaie ici aussi de « dire la même chose au même moment », mais n'y parvient que d'un point de vue sémantique : la congruence achevée ici est n'est pas littérale (de dicto), mais uniquement référentielle (de re). Pourtant, de par son anticipation, le coénonciateur arrive à communiquer effectivement et à « prouver » par sa performance interactive qu'il partage le savoir du sujet en question.

La structure bipartite fournissant la base pour la prévisibilité d'un deuxième élément peut aussi être une phrase comparative, $c f$. (6). La jonction dont se sert la coéonciatrice pour initier est ici encore la complétude de la première composante de la comparaison ("plus sur A »).

(6) Ex. Müller (1995 : 72)

$01 \quad$ M1: la prof savait puisque je lui avais parlé j'étais arrivé en retard et elle savait que

02 \&j'étais euh je n'étais pas français (.) à ce moment là euh (.) je quand mes- dans

03 \&mes- dans mes devoirs ELle (.) elle me sanctionnait beaucoup PLUS sur les fautes

04 \&d'orthogr[aphe-

$05 \Rightarrow$ W3: $\quad$ [que sur le contenu,

06 M1: que sur le contenu.

Notre dernier exemple pour illustrer l'importance des jonctions syntaxiques pour les co-constructions montre un type qu' on pourrait appeler « surfer sur le tour de parole d'un autre ». En (7), le coénonciateur (M1) attend jusqu'à une frontière syntaxique majeure. Quand elle est atteinte, il se sert de " l'espace d'opportunité » qui s'ouvre ici pour pouvoir initier une contribution qui s'accroche au syntagme nominal précédent et ajoute un commentaire bref qui ne semble pas gêner le locuteur en cours à continuer immédiatement son tour. Ainsi, les deux interlocuteurs coopèrent pour se servir du bout d'espace libre qui s'est ouvert à la jonction syntaxique. La coénonciation - qui a l'intonation d'une phrase incise - est ainsi parfaitement intégrée comme une parenthèse grammaticale ${ }^{(13)}$ :

(7) Ex. Müller (1999: 181)

$01 \quad$ M2: pour l'académie française y a un truc (.) c'est que les mecs qui sont à l'académie française

02 \&quand tu lis les noms sur la liste c'sont des types qui écrivent dans un français excellent

03 \&au point de vue eu:h litteraire-=

$04 \Rightarrow \mathrm{M} 1$ : =et c'est EUX qui définissent la norme.=

$05 \quad$ M2: =mais au point de vue de nouveauté de la langue (.) que dalle

(13) Jeanneret (1999: 268) appelle ce type, où il y a un «locuteur second », qui ne fait qu'insérer une précision dans le discours d'un « locuteur principal», une «coénonciation par ajout parenthétique ». 


\subsection{Réparations}

L'occurrence de pauses, d'hésitations, de recherches lexicales et d'interruptions de toutes sortes dans la progression émergente des énoncés mettent en jeu la coordination et la continuation de l'interaction et jouent un rôle important dans la genèse interactive des coénonciations. Ce n'est pas seulement entre des tours de parole complets que les participants ont le horror vacui et tendent à éviter des pauses entre des tours La tendance à éviter des lacunes se trouve aussi à l'intérieur des tours (et à l'intérieur des constructions syntaxiques) déjà initiés, mais visiblement encore incomplets. Des troubles dans l'émergence invitent à la continuation par coénonciation ${ }^{(14)}$. L'étude des données d'un corpus considérable, rassemblées en vue d'une analyse empirique de la coénonciation en français (Jeanneret 1999) nous semble soutenir cette thèse : la grande majorité des exemples de coénonciation se groupe dans la catégorie des réparations, qui répondent à des troubles d'émergence interrompue.

Pour la « coénonciation en réparation », Jeanneret (1999) propose une analyse en trois tours/trois positions séquentielles : a) un premier tour de l'interlocuteur originaire contenant la « source du trouble » motivant la coénonciation; b) un deuxième tour présentant l'unité coénoncée par un récepteur / interlocuteur suivant ; c) un troisième tour de l'interlocuteur originaire ratifiant - ou rejetant - la réparation coénoncée. Pour illustrer cette analyse, $c f$. son exemple :

(8) Ex. Jeanneret (1999: 265)

$\begin{array}{lll}01 & \text { A: } & \text { la fin du match devient dure euh: } \\ 02 & \text { B: } & \text { houleuse } \\ 03 & \text { A: } & \text { houleuse tout à fait }\end{array}$

Il est évident que l'origine du trouble est analysée et interprétée par le récepteur/coénonciateur. Dans le cas présent, il ou elle interprète l'hésitation (dure euh:, 01) comme signe d'une recherche lexicale, poursuivie pour trouver un terme qui est plus adéquat que le terme déjà trouvé. Le caractère lexicalement plus spécifié et plus élaboré de ce terme (houleuse) est évidemment le résultat d'une telle analyse du trouble perçu ${ }^{(15)}$.

Employant une terminologie différente, Lerner (1996) conçoit les troubles qui peuvent obstruer l'émergence ultérieure d'une construction plutôt comme une ressource pour le récepteur - et coénonciateur potentiel - de pouvoir prendre la parole : les lacunes dans l'avancement d'un tour de parole font surgir un « espace d'opportunité » («opportunity space ») et «provide the possibility for another participant to produce an opportunistic completion for the turn constructional unit » (Lerner 1996 : 261). La complétion « opportuniste » est un dispositif servant à la réalisation de toute une diversité de relations interactives. Dans des séquences de recherche lexicale, par exemple, elle peut se faire au service d'un travail de «positionnement» ( «alignment»). Le coénonciateur ne se limite pas à faire avancer et compléter la construction en cours, mais « s'aligne »: " For example, early opportunistic completion of a word search is a device that can be used to initiate or sustain a special alignement with a speaker, one of story consociateship or association co-membership rather than recipientship » (Lerner $1996: 263)$. 


\section{Présentation et discussion des données de la LUTINE}

\subsection{Savoir indépendant}

Nos premiers exemples proviennent d'une réunion avec quatre participants, tous membres d'un groupe proféministe lyonnais, qui discutent de la préparation d'un tract pour représenter les idées du groupe à un plus grand public. Dans ce cercle, la discussion ressemble un peu à un débat entre intellectuels qui sont en train de co-élaborer une identité discursive commune. Un pas dans cette direction consiste dans le fait que tous les participants sont d'accord pour éviter les généralisations grossières caractérisant beaucoup de tracts unifiés et pour affirmer qu'une telle unification annihile, c'est-à-dire ferait disparaître les points de vue diversifiés pouvant coexister à l'intérieur d'une position commune.

Dans la réunion, on trouve beaucoup de chevauchements qui sont pourtant pour la grande majorité de nature coopérative et non pas compétitive : ainsi, dans l'extrait suivant ( $c f$. par exemple 04), AN s'insère en chevauchement. Mais en reprenant et reproduisant d'une manière affirmative des éléments lexicaux et syntaxiques du tour de son prédécesseur, son intervention se révèle rapidement, c'est-à-dire dès son début (trois trois apports (...) trois apports, 04), comme l'interprétation affiliative et consensuelle du tour émergent de MC qui est toujours en cours :

(9) Ex. Klaeger (MECS, 1170-1179)

$01 \mathrm{MC}$ : =moi je oui trois avis différents je vois pas pourquoi [li faudrait (...) ben peut-être qu'ils

02 AN: [ben (...) oui'fin mais

03 MC : \&soient forcément différents [qu'ils se complètent

04 AN : $\quad$ [trois trois apports (..) trois apports qui se complètent et qui

05 \&sont euh=

06 MC: =je trouve que c'est vachement mieux qu'essayer

07 \&plutôt de faire [un truc global [qui à chaque fois

08 AN : [oui [moi je suis d'accord

09 MC : \& [en fait euh annihile euh

$10 \Rightarrow \mathrm{AN}: \quad \&[\mathrm{hmhm}($.$) annihile euh l'ensemble des euh ouais=$

$11 \quad M C$ : =ouais ouais

L'affiliation et le consentement thématique entre les interlocuteurs dans la matière en question s'expriment ici aussi par la coopération lexico-syntaxique qu'on a décrite ci-dessus par les termes « savoir indépendant» et " chevauchement accompli », $c f$. l'annihile de AN en 10, qui arrive précisément en parallèle avec celui prononcé par MC. L'occurrence du verbe annihiler, qui peut de prime abord apparaître étonnante, a tout de même sa prévisibilité ici : il constitue un contraste sémantique, une « antonymie », lexicale et locale, par rapport au pré-

(14) $C f$. Jefferson $(1984: 37):$ «Hitches generate recipient activities ». Ces activités de réception peuvent être aussi du genre nonverbal. Il a été observé et décrit ( $c f$. Goodwin/Goodwin 1992) qu'un récepteur présent qui n'a pas regardé jusqu'ici lève son regard pour focaliser l'interlocuteur au moment où celui-ci s'engage dans une recherche lexicale et le suit du regard pendant cette recherche jusqu'à sa fin.

(15) La collaboration syntaxique et lexicale pour négocier et trouver des termes adéquats peut être un processus continu, surtout quand il s'agit de s'accorder sur une expérience commune aux participants, $c f$. Mondada (1999). 
décesseur-se compléter. Le contraste se déploie, dans son émergence linéaire et séquentielle, le long d'une construction syntaxique comparative à deux temps : la première composante articule et propose une option préférée, qui est rapidement acceptée, $c f$. AN qui reprend une partie du tour en cours de MC (trois apports qui se complètent, 04/05) ${ }^{(16)}$. La deuxième contient une option non préférée correspondante : un truc global qui (...) annihile (07/09). Si l'on représente cette émergence séquentielle par un schéma simplifié, on reconnaît la cohérence de la gestalt, un chiasme par le croisement des termes, dont la clôture est atteinte à l'arrivée du verbe annihile :

\section{1. trois apports différents qui se complètent}

2. un truc global qui annihile (les différences)

Par le chevauchement accompli sur annihile s'expriment la rapidité et la précision d'une compréhension « parfaite »- le récepteur/locuteur suivant reconnaît et identifie les idées du locuteur en cours avant même que celui-ci n'ait fini de les mettre en mots et peut ainsi signaler qu'il est sur la même longueur d'onde. On remarquera aussi qu'après la découverte de l'unanimité manifestée par l'anticipation, aucun des deux interlocuteurs ne considère plus nécessaire de continuer et compléter ultérieurement la phrase initiée au-delà de ce point. Ceci permet l'interprétation qu'on peut laisser tomber un sujet, qui a été éclairci suffisamment à ce point précis entre les participants.

On peut assumer dans un cas pareil qu'il y une correspondance entre la microprocédure, la pratique interactive du savoir indépendant déjà décrite, et l'arrièrefond du (macro-)savoir idéologique d'un groupe libertaire dans lequel on essaie de tenir compte de la pluralité des opinions et de l'expression et où l'avis de l'individu compte autant qu'un quelconque avis du groupe.

\subsection{Collectivités}

Dans les conversations plurilocuteurs des réunions, un interlocuteur donné se trouve en général en face de tout un groupe de co-récepteurs/prochains locuteurs possibles. La co-appartenance à un groupe peut avoir des «conséquences procédurales » $\left(c f\right.$. Schegloff 1991) ${ }^{(17)}$. Ces conséquences se montrent dans un élargissement de la participation dans des scènes, où les participants s'adressent mutuellement en tant que membres du même groupe :

La pratique d'adresser des récepteurs en tant que membres d'un groupe et la pratique de répondre pour le groupe créent des possibilités pour élargir l'unité de participation et la transforment d'un mode individuel envers un mode plurilocuteur. Le groupe devient une unité importante dans l'organisation de l'interaction.

(Lerner $1993: 214$; trad. F. E. M.)

(16) On remarquera la complexité et la rapidité de l'intercompréhension en évidence ici : pour pouvoir reprendre $\mathrm{MC}$ tel qu'il le fait, AN doit comprendre (tout en parlant en chevauchement avec lui) ce que dit MC.

(17) Dans sa discussion des « membership categorization devices » introduites par Sacks, Schegloff (1991) insiste sur le principe qu'il faut chaque fois justifier le choix d'une catégorie en démontrant que la catégorie choisie a de l'importance pour les participants euxmêmes et qu'elle entraîne des « conséquences procédurales » pour l'organisation socio-séquentielle de leur interaction. 
L'exemple suivant documente une co-construction dans une séquence de recherche lexicale. Le «travail de dénomination » (Blanche-Benveniste 1984) qui se manifeste ici montre les traits esquissés qui caractérisent l'interaction du groupe dans ses réunions, en particulier la co-participation de plusieurs interlocuteurs à l'intérieur d'un seul tour de parole. Cette co-participation est évoquée souvent dans les actes de nomination, quand il s'agit de nominer et en même temps catégoriser ${ }^{(18)}$ des personnes ou des objets d'un point de vue des intérêts du groupe, de sa «version du monde ». Ces actes introduisent la pertinence d'un point de vue collectif et de la façon dont il est partagé par les membres présents. Ils montrent en même temps des facettes de la construction et de la négociation de l'identité du groupe dans l'interaction.

En 01, RA propose de confier une tâche de négociation - délicate pour le groupe - à une personne (pierre), qui est avocat et connu par les membres du groupe présents. Ceci crée la pertinence pour les récepteurs présents de parler et de répondre à cette proposition en tant que membres du groupe concerné. RI, en 04, réagit à la proposition et initie une réponse, tout en se mettant à formuler un jugement à propos de pierre et à indiquer le rapport actuel de celui-ci avec le groupe :

(10) Ex. Klaeger (R7, 3073-3081)

\begin{tabular}{|c|c|}
\hline RA & [à la limite eu:h (.) c'est peut-être un truc à demander à pierre en \\
\hline 02 & \&apparté à \\
\hline SY & $\mathrm{hm} \mathrm{hm}$ \\
\hline 04 & plus quoi \\
\hline $\begin{array}{l}05 \\
06\end{array}$ & $\begin{array}{l}\text { alors pierre j'ai un (.) j'aurais plutôt l'impression qu'il veut plus du tout entendre } \\
\text { \&parler de: nous en tant que: enfin [au: à l'intérieur [de son bou:- non non plutôt }\end{array}$ \\
\hline $07 \Rightarrow \mathrm{RA}$ & [en tant qu'amis/ \\
\hline & [comme clients/ \\
\hline
\end{tabular}

La réponse de RI montre dès son début - $c f$. la rupture entre le premier départ et le départ réorganisé en 05 (pierrej'ai un (.) j'aurais plutôt l'impression) - plusieurs marques d'un travail de formulation difficile ${ }^{(19)}$. Ces difficultés manifestes culminent quand il s'agit de formuler le rapport entre pierre et nous $-c f$. les hésitations/allongements de syllabe, les autocorrections et reformulations (de: nous en tant que: enfin au: à l'intérieur, 06). La recherche d'un terme et la façon dont cette recherche est démontrée aux interlocuteurs invitent l'intervention de deux coénonciateurs. (Il est bien possible que la deuxième intervention, qui propose une catégorisation opposée, soit provoquée par la première). Dans les deux cas, les coénonciateurs ne proposent pas une unité lexicale seule, mais un syntagme, approprié pour établir un gestaltschluss de la trajectoire en cours. Dans

(18) Pour une description détaillée de l'auto-catégorisation du groupe, $c f$. Klaeger (2007).

(19) Pour le travail de formulation et de reformulation, $c f$. Gülich (2008). Ce travail laisse des traces linguistiques dans le discours oral : «Un énoncé dans une conversation n'est sûrement pas un produit fini, confectionné et optimé. Le producteur/interlocuteur oral ne peut « raturer » et enlever - comme dans un texte écrit - toutes les corrections, faux départs, reformulations etc., avant de le présenter à son récepteur co-présent. Au contraire, le récepteur participe à la genèse du discours, même s'il n'est, en ce moment, qu'un récepteur silencieux » (Gülich 2008 : 351 ; trad. F.E.M.) Mais en général, le récepteur, ainsi que c'est le cas ici, en face de phénomènes de la sorte, ne reste pas silencieux. 
les deux cas il est évident aussi que ces syntagmes ne dépassent pas, en longueur, un format minimal qui permette ce gestaltschluss. Le rôle de l'appartenance au même groupe qui se reflète dans une co-participation aux actes d'auto-description et d'auto-catégorisation se montre également dans l'exemple suivant. Les membres du groupe sont ici encore en train de préparer leur procès imminent. Ils réfléchissent comment, c'est-à-dire sous quelle apparence, ils pourront se présenter au public de ce procès.

(11) Ex. Klaeger (R1, 709-723)

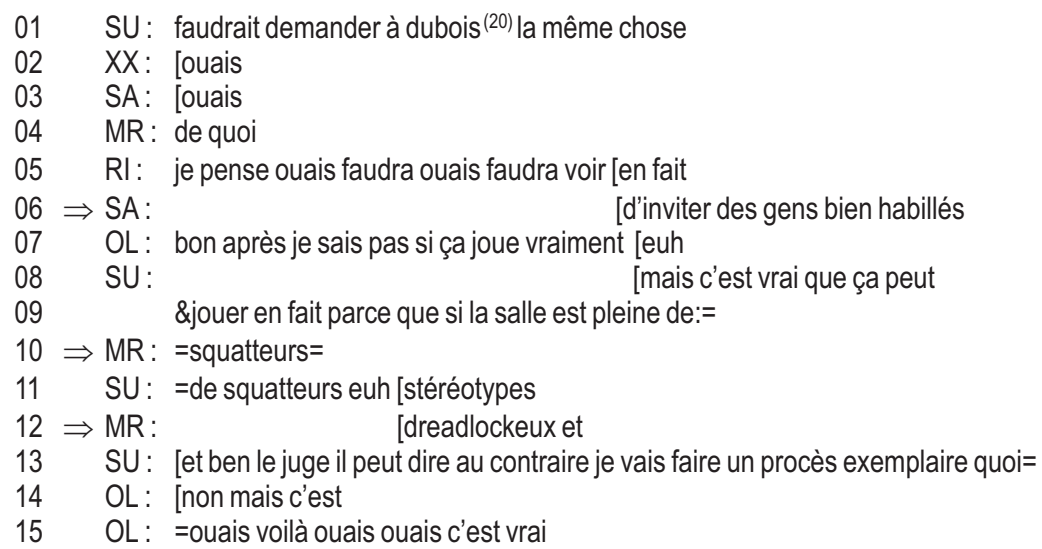

Le thème de l'auto-catégorisation du groupe - vu par les yeux d'un juge imaginaire - est introduit séquentiellement par une paire contrastive, une antonymie locale de deux termes d'une collection de catégories sociales qui peuvent s'évoquer mutuellement : le premier élément ici est la catégorie des gens bien habillés, $c f$. la co-construction de SA en 06. Il évoque-c'est-à-dire projette et rend prévisible - le contraste d'avec le deuxième élément, la catégorie opposée, qui est en même temps la catégorie auto-référentielle : les squatteurs de MR en 10, les squatteurs euh stéréotypes de SU en 11, les (squatteurs) dread lockeux de MR en 12. Les deux derniers termes - les adjectifs stéréotypes et dreadlockeux-qui sont produits en chevauchement accompli ne sont pas lexicalement identiques, comme c'est le cas idéal de la coïncidence parfaite manifestée par le « même mot au même instant », mais se réfèrent à la même qualité ${ }^{(21)}$.

Ainsi, on développe, en coélaboration, de manière brève et rapide il est vrai, un auto-portrait, une image identitaire du groupe vue de l'extérieur. Evoquer ce thème de l'autoportrait du groupe semble évoquer aussi un changement dans le cadre de participation : on notera la densité de la co-participation de cette scène, dans laquelle six interlocuteurs/interlocutrices s'engagent. Entre la matière

(20) Dubois (nom changé) est l'avocat du groupe. la même chose se réfère à un autre cas où un avocat avait souligné l'importance d'inviter des personnes «cleans » à venir soutenir les squatteurs lors d'un procès.

(21) Comme le note Klaeger (2007 : 147) : « les dread locks fonctionnent ici comme un pars pro toto dans la description. Cette coiffure est désignée comme emblématique parce que plus remarquable que, par exemple, les cheveux tondus courts qui sont au moins aussi répandus chez les squatteurs. Son évocation est surtout représentative d'un prototype (dans le sens de représentant exemplaire) de squatteurs [...]». 
dont on parle ici et l'organisation interactive (et surtout la manière de co-participation), il nous semble exister un rapport symbolique qui n'est pas aléatoire.

\subsection{Partage de l'espace locutoire ("sharing the floor»)}

Nous nous sommes déjà référés, dans l'introduction, à l'argumentation de Coates (1994), selon laquelle il existe, à coté de 1'organisation des tours de parole telle qu'elle a été décrite par Sacks et al. (1974), d'autres formes, des stratégies de prise de parole qui sont caractérisées surtout par une grande densité de chevauchements et par des collaborations syntaxiques. Peut-on, dans nos données, trouver des scènes d'interaction qui correspondent à cette description ? Il y a des passages, par exemple dans la réunion MECS dont on a déjà parlé, où la CS se prolonge et englobe plusieurs tours de parole à la fois. Nous avons un tel exemple en (12), où les trois mêmes interlocuteurs reviennent à un sujet déjà introduit quelques minutes auparavant ( $c f$. l'exemple (9) commenté supra). Ils construisent d'une manière consensuelle un argument ensemble. Celui-ci est indiqué au début du tour par l'interlocuteur MC, qui exprime sa préférence pour les textes individuels ( $c f$. 01/02) :

(12) Ex. Klaeger (MECS, 1471-1479)

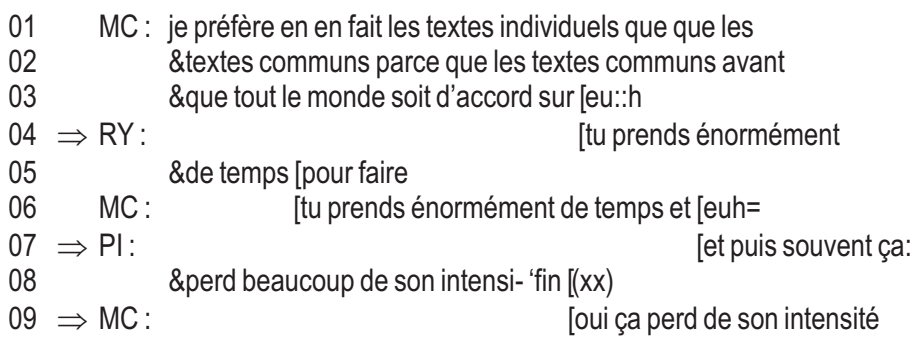

Il est évident qu'ici encore, ce sont les troubles dans l'émergence ultérieure des énoncés (hésitations en 03 et 06,1 'amorce d'une reformulation en 08 ) qui fournissent l'espace interlocutif où les coénonciateurs RY et PI initient leurs énoncés. Comme le formule Jefferson (1984 : 37) : «Hitches generate recipient activities ».

Les tours des deux co-constructeurs RY et PI contribuent au tour dominant qu'ils « alimentent». MC reste l'interlocuteur dominant qui réclame et reconquiert chaque fois le droit à son tour. Il traite chaque fois (06 et 09) les contributions comme « accountable», i.e. il les ratifie, les prend en compte et se comporte ainsi en tant qu'interlocuteur dominant. On voit donc malgré le caractère continu de la CS que le principe de « un locuteur à la fois » est néanmoins respecté.

Dans le prochain exemple, nous n'avons pas une CS dans le sens strict d'une « collaborative sentence completion » puisqu'il ne s'agit pas d'une construction syntaxique qui est achevée en commun. Il est évident pourtant qu'il y a là CS. Celle-ci n'est pas un fait ponctuel, mais une modalité d'interaction soutenue qui englobe des continuations d'un tour de parole venant de plusieurs locutrices différentes. On pourrait parler ici avec Coates d'un «sharing of the floor [which] is symbolic of intimacy » (Coates $1994: 188)$ : 
(13) Ex. Klaeger (POILS, 22-44)

$01 \quad$ EL : ouais ouais parce que (.) je vois t'as t'as les poils au bout des pieds là (.) \&moi je connais des fill[es qui se les enlèvent ces poils-là (.) ou juste euh sur

L'activité descriptive ici, un listing prolongé, est d'abord celle d'une seule locutrice (EL). Elle se sert, en développant son tour (et son tour d'horizon sur les poils) d'un seul schéma syntaxique : un présentatif existentiel personnel ( $t u$ as $X_{1} \ldots$, tu as $X_{2} \ldots$, tu as $X_{3}$.) qui adresse la parole aux récepteurs ${ }^{(22)}$ et les invite à la co-construction par l'addition d'autres $X$ convenables - des expansions du tour qui emploient le même format ${ }^{(23)}$. La construction permet en même temps de «maintenir un topic» («topic retaining »), ici la catégorie $\mathrm{X}(\mathrm{X}=$ les poils $)$, et de le «varier» («topic shifting ») - ici détailler et énumérer ses manifestations particulières $\mathrm{X}_{1}, \mathrm{X}_{2}$, etc.

EL clôt son tour en 07 avec un commentaire évaluatif et la particule de clôture quoi. D'autres participants $(\mathrm{SU}, \mathrm{RI})$ rajoutent des expansions, se joignent à l'activité énumérative initiée, et complètent ce tour d'horizon sur les poils. SU reprend le même schéma syntaxique et «taille» ses contributions de façon à être reconnaissables en tant que continuations du tour de parole initié.

La coénonciation transmet ici la familiarité de rapports et l'orientation thématique partagée entre les participantes : d'une part, la coénonciation est le produit d'un tel alignement dont il faut présupposer l'existence ; d'autre part, c'est seulement par la coénonciation continuée qu'une telle orientation peut être manifestée et réalisée en tant qu'identité interactive et discursive du groupe.

\subsection{Récit conversationnel}

En général, les récits conversationnels ont un «motif local» (« local occasionment », Jefferson (1979) qui les ancre dans l'interaction précédente. Ce motif est évident dans l'exemple (15), cf. lignes 01-02. Le récit est ainsi « topically

(22) Pour l' " embedded addressing », $c f$. Lerner (1993: 225) : « Addressing can be embedded in person reference by employing the "recipient proterm" you ".

(23) Le format du listing prolongé est souvent utilisé dans l'enseignement scolaire pour éliciter la co-participation de plusieurs participants dans la construction continuée d'un seul tour de parole, $c f$. Lerner (1995). Il est en même temps un exemple illustratif pour l'expansion incrémentale des TCUs. Pour le rôle de la syntaxe incrémentale dans 1'AC, $c f$. par exemple Auer (2007), Horlacher (2007), Mondada (2007), Schegloff (1979). 
coherent » et fonctionnel pour le discours dominant de la réunion, où l'on parle de la nécessité de fermer à clé les appartements dans le squat ${ }^{(24)}$.

Le récit est préfiguré par une préface ( « résumé », " abstract») qui projette le déroulement des évènements à raconter dans la suite et en indique leur résultat final (cf. il nous a taxé une clef, 07). Dans notre exemple, la préface est congruente presque littéralement avec la pointe conclusive du récit, le dénouement ("résolution ») dans ses deux versions (cf. et le gardien (xxx) la clef, 12 ; et il a piqué la clef/ putain, 13).

(14) Ex. Klaeger (R2, 765-793)

01 RO : mais moi je pense maintenant vaut mieux commencer à fermer les

02 \&portes (...) parce que si le gardien revient euh=

03 VE: $=\mathrm{hm}=$

$04 \mathrm{RI}$ : $=(\mathrm{x})$ mais parce qu'en plus voilà voilà c'est faut comprendre qu'il $\mathrm{y}$ a un gardien

05 RO: $h m$

$06 \mathrm{Rl}$ : qui vient faire le ménage/ (.) et eu:h il est quand même du genre a: a rentrer dans les

07 \&apparts s'il les trouve ouverts ou eu:h il nous a taxé une clef (.) au moment de la du

08 \&week-end de la gryffe il y avait (.) on avait ouvert c'était l'appart (.) en bas de

$09 \&$ \& (chez nous (.) ou enfin l'appart ?) où il y a amélie et gilles et ito maintenant (.)

10 \&et eu:h on avait laissé pendre la clef/à une ficelle pour pas ( $\mathrm{xxxxx})$

11 \&arrivent $(\mathrm{x})$ gens ( $\mathrm{xxxxx}$ ) puissent rentrer et sortir sans se faire

12 \&chier/ (..) [et le gardien (xxx) la clef/

$13 \Rightarrow V E$ : $\quad$ [et il a piqué la clef/ putain

Dans la trajectoire du récit, l'émergence du dénouement est retardée par une pause du narrateur en 12. Cette pause a lieu au site d'une jonction structurelle importante du récit et sert à détacher et mettre en relief la pointe de la clôture. Ce ritardando, à l'état manifestement encore incomplet du récit en cours, provoque la coénonciation de VE qui réalise un gestaltschluss du récit en simultanéité avec le narrateur. VE s'installe ainsi en tant que co-narrateur qui connait les évènements racontés, mais aussi le détail de la forme narrative d'un récit qui a probablement déjà circulé dans le groupe. Le récit touche aussi l'identité du groupe - le protagoniste est qualifié en tant qu'ennemi du groupe qui pourrait devenir dangereux pour les intérêts du squat. Par sa formulation "fortifiée » (putain, 13), VE documente qu'il partage cette évaluation collective du gardien.

\section{5. «Actions imaginaires »}

Une forme de plaisanterie qui produit un changement de modalité dans le style sérieux des réunions, se manifeste dans ce que Klaeger (2007) appelle des « actions imaginaires ». Il s'agit d'actions virtuelles du groupe que l'on ne pense pas réaliser, mais dont on parle, qu'on dépeint d'une manière théâtrale et parodique : «Ces actions imaginaires sont des petites mises en scène non sérieuses, qui reflètent le point de vue politique et idéologique du groupe d'une manière exagérée et déformée » (Klaeger $2007: 223$ ).

(24) «A story is "triggered" in the course of turn-by-turn-talk. That is, something said at a particular moment in conversation can remind a participant (speaker or hearer) of a particular story, which may or may not be "topically coherent" with the talk in progress " (Jefferson $1978: 220)$. 
L'exemple suivant d'une telle action imaginaire est tiré de la réunion déjà citée, où les membres du groupe se préparent à la convocation pour un procès imminent. Lors de cette audience, le juge décidera si l'expulsion de l'immeuble occupé doit être immédiate ou s'il accorde un délai. C'est après une longue discussion à ce propos que RO présente un discours qu'il imagine donner devant le juge. Les premiers pas qui transposent la modalité du débat sérieux envers le jeu ironique et théâtral de l'action imaginaire se trouvent dans l'énoncé métadiscursif ben moi je vous avertis en 01 et la collaboration lexico-syntaxique entre RO et RI en $02 / 03$ :

(15) Ex. Klaeger (R1, 661-672)

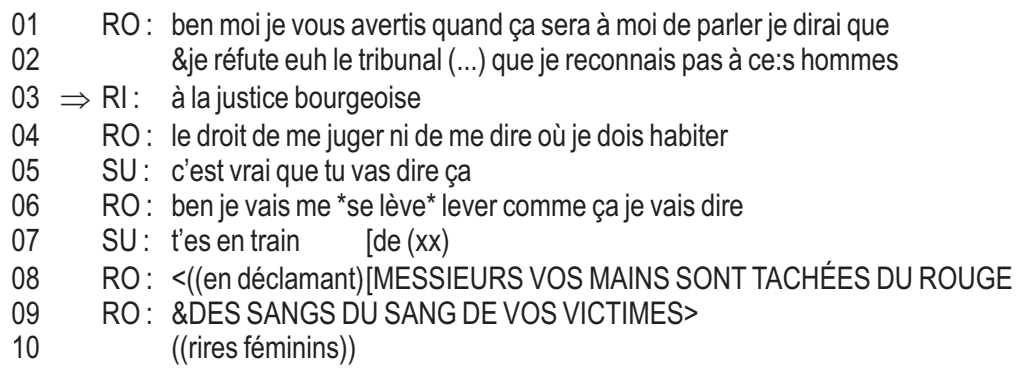

Déjà la première formulation de RO (je dirai que je réfute euh le tribunal, 01/02) contient une tournure qui appartient au registre contestataire de la gauche des temps passés, qui n'est plus en usage dans notre groupe de squatteurs. La tournure renvoie à un emploi ironique et contextualise un emploi de citation de la rhétorique révolutionnaire grandiloquente. Par la suite, dans un procédé d'élaboration et de reformulation, l'interlocuteur hésite dans la progression de son discours et ajoute une deuxième formulation, qui élabore la première (que je reconnais pas à ce:s hommes, 02) ${ }^{(25)}$. Ici, il lui manque le mot clé pour désigner l'ennemi, il utilise donc l'expression passe-partout ce:s hommes. C'est déjà à ce moment que RI rentre dans l'amorce du jeu ironique. Christmann, dans son travail sur un groupe d'écologistes, explique ces réactions immédiates par « l'arrièreplan du savoir spécialisé écologique et de l'idéologie commune " (Christmann 1996 : 62 ; trad. S.K.). Il en va de même pour les squatteurs qui utilisent leur culture anarchiste. En fait, RO cite ici, presque littéralement, des passages d'un dialogue entre l'anarchiste Emile Henri et un juge lors de son procès en 1894 (dialogue présenté dans Maitron (1992), que les interactants ont tous lu). La reformulation (à lajustice bourgeoise, 03) de RI contient également une tournure qui fait partie de la rhétorique de la gauche traditionnelle. Elle renforce les éléments ironiques déjà présentes, les " ratifie » et co-construit le changement déjà initié envers la modalité ironique de l'action imaginaire en progression. En 04, RO continue et termine « sa » construction initiée en 02 .

(25) Pour l'importance des procédés de reformulation dans la genèse d'un texte spontané, $c f$. Gülich (2008). Une propriété linguistique des reformulations consiste dans le fait qu'ils retiennent des éléments identiques par rapport à leur prédécesseur(s), mais les transforment en apportant des éléments nouveaux. Dans notre exemple, on peut bien voir que l'interlocuteur RI prolonge 1'activité des reformulations et qu'il y a ainsi un rapport entre élaboration sémantique du texte et CS. 


\section{Conclusion}

Au début de cet article, nous avons proposé d'étudier le rôle et les fonctions des CS dans l'interaction verbale d'un groupe donné et nous avons posé la question de savoir si l'appartenance à un même groupe a un effet sur l'emploi des CS.

La co-appartenance à un groupe a des "conséquences procédurales » (Schegloff 1991) dans l'interaction et nous semble favoriser l'occurrence de CS. Les procédés du « within-turn-talk » et de la coénonciation permettent aux locuteurs de «s'aligner », de prendre une position envers le déploiement ultérieur du tour de parole en cours et toujours en émergence. Les positions observées étaient, dans la grande majorité des CS analysées, celles de la concordance, c'est-à-dire des interprétations affiliatives et consensuelles du tour émergent : les locuteurs marquent l'écoute attentive par une analyse et une interprétation située qui se réalise online et qui n'attend pas la fin de la trajectoire; ils montrent et marquent le parallélisme d'associations, d'idées et du savoir qu'ils partagent avec le locuteur en cours, et qui mène au même résultat d'une formulation identique ou semblable. Le gestaltschluss achevé par une coénonciation peut également se rapporter à un enchaînement argumentatif pour renforcer l'évidence d'une conclusion, "prouvée " par le fait que ce sont plusieurs participants qui arrivent au même moment au même résultat. Généralement, les CS et les chevauchements accomplis représentent un ensemble important de moyens qui servent à se signaler la concordance située.

Les CS ont un aspect temporel : bien se comprendre, c'est aussi se comprendre en rapidité. Le locuteur suivant reconnaît et identifie les idées émergentes du locuteur courant avant même - ou en même temps - que celui-ci n'ait fini à les mettre en mots. En plus, dans des situations pluri-locuteurs telles quelles se présentent dans nos données, on économise dans la «machinerie» des prises de parole en s'insérant par coénonciation plus directement dans le tour en cours et en préférant ainsi des interactions courtes et rapidement enchâssées dans la progression du discours.

Dans l'interprétation de nos exemples, on a observé et commenté l'aspect de la projection qui rend possible l'anticipation et le «precision timing » du placement des continuations des tours de parole en émergence.

L'appartenance au même groupe n'a sans doute pas une incidence uniforme et invariable sur l'interaction. Mais elle peut être pertinente et manifestement présente avec des conséquences procédurales, quand les participants s'adressent mutuellement en tant que membres du même groupe, évoquent la pertinence des points de vue communs et des intérêts spécifiques du groupe et de sa vision du monde. C'est dans les scènes de ce genre, qui font apparaître des facettes de la construction de l'identité du groupe, située dans une interaction particulière courante, que le mode individuel de participation « tour de parole suit tour de parole » $-\mathrm{a}$ tendance à se transformer vers un mode pluri-locuteurs où les frontières des tours sont plus perméables et les pratiques co-constructrices plus fréquentes. Dans ces scènes, le jeu des projections est imprégné par la co-appartenance au groupe, $c f$. notamment les exemples analysés de l'auto- et hétéro-catégorisation des squatteurs. Ici on quitte la syntaxe et entre sur le plan sémantique et pragmatique. Analyser la construction des tours de parole sur ce plan : un travail qui reste à faire. 


\section{Références}

AUER, P. (2007) : «Syntax als Prozess », in H. Hausendorf(éd), Gespräch als Prozess. Linguistische Aspekte der Zeitlichkeit verbaler Interaktion, 95-142. Tübingen, Narr.

- (2002): «Projection in interaction and projection in grammar », InList, 33, 39 p.

- (1996) : « On the Prosody and Syntax of Turn-continuations », in E. CouperKuhlen et M. Selting (éds), Prosody in conversation, 57-100. Cambridge, Cambridge University Press.

Blanche-BenVEniste, C. (1984) : «La dénomination dans le français parlé : une interprétation pour les "répétitions" et les "hésitations" ", Recherches sur le Français parlé, 6, 109-130.

Christmann, G. B. (1996) : « Die Aktivität des "Sich-Mokierens" als konversationelle Satire. Wie sich Umweltschützer/innen über den "Otto-Normalverbraucher" mokieren », in H. Kotthoff (éd), Scherzkommunikation. Beiträge aus der empirischen Gesprächsforschung, 49-80. Opladen, Westdeutscher Verlag.

COATES, J. (1994) : «No gap, lots of overlap : Turn-taking patterns in the talk of women friends ", in D. Graddol, J. Maybin et B. Stieter (éds), Researching Language and Literacy in Social Context, 177-192. Clevedon, Open University.

Goodwin, C., Goodwin, M. H. (1992) : « Context, Activity and Participation », in P. Auer et A. di Luzio (éds), The Contextualization of Language, 77-99. Amsterdam, Benjamins.

GÜLICH, E. (2008) : « Reformulierungen », in I. Kolboom, T. Kotschi et E. Reichel (éds), Handbuch Französisch : Sprache-Literatur - Kultur-Gesellschaft, 359-367. Berlin, Schmidt.

- (1986) : «L'organisation conversationnelle des énoncés inachevés et de leur achèvement interactif en situation de contact », $D R L V, 34 / 35,161-182$.

HORLACHER, A.-S. (2007) : «La dislocation à droite comme ressource pour l'alternance des tours de parole : vers une syntaxe incrémentale », Tranel, 47, 117136.

JACOBY, S., OCHS, E. (1995) : « Co-Construction : An Introduction », Research on Language and Social Interaction, 28/3, 171-183.

JEANNERET, T. (2001) : «Vers une respécification de la notion de coénonciation : pertinence de la notion de genre », Marges Linguistiques, 2, 81-94.

- (1999) : La coénonciation en français. Approches discursive, conversationnelle et syntaxique, Berne, Lang.

JefFerson, G. (1986) : « Notes on latency in overlap onset », Human Studies, 9, 153-183.

- (1984) : « Notes on some orderliness of overlap onset», in V. d'Urso et P. Leonardi (éds), Discourse analysis and natural rhetorics, 11-38. Padova, Cleup.

— (1979) : «A Technique for Inviting Laughter and its Subsequent Acceptance Declination ", in G. Psathas (éd), Everyday Language : Studies in Ethnomethodology, 79-96. New York, Irvington Press.

- (1978) : « Sequential aspects of storytelling in conversation », in J. Schenkein (éd), Studies in the organisation of conversational interaction, 219-248. New York, Academic Press. 
— (1973) : «A case of precision timing in ordinary conversation : Overlapped tag-positioned address terms in closing sequences », Semiotica, 9, 47-96.

KALLMEYER, W. (1999) : " Others' Inserts in an ongoing turn. Some Sequential, Grammatical and Rhetoric observations ", in: E. Rigotti (éd), Rhetoric and Argumentation, 255-268. Tübingen, Narr.

KLAeger, S. (2007) : La Lutine. Portrait sociostylistique d'un groupe de squatteurs à Lyon, Paris, L'Harmattan.

LERNER, G. H. (1996) : «On the "semi-permeable" character of grammatical units in conversation : conditional entry into the turn space of another speaker », in E. Ochs, E. A. Schegloff et S. Thompson (éds), Interaction and Grammar, 238-276. Cambridge, Cambridge University Press.

- (1995) : «Turn design and the organization of participation in instructional activities », Discourse Processes, 19/1, 111-131.

- (1993) : «Collectivities in action: Establishing the relevance of conjoined participation in conversation », Text, 13/2, 213-245.

- (1991) : « On the Syntax of Sentences-in-Progress », Language in Society, 20/3, 441-458.

MAitron, J. (1992) : Ravachol et les anarchistes, Paris, Gallimard.

MONDADA, L. (2007) : "L'interprétation online par les co-participants de la structuration du tour in fierien TCUs : évidences multimodales », Tranel, 48, 7-38. — (1999) : "L'organisation séquentielle des ressources linguistiques dans l'élaboration collective des descriptions », Langage et société, 89, 9-36.

MÜlLER, F. E. (1999) : "Turn-taking und Thematisierung im Französischen », in A. Redder et J. Rehbein (éds), Grammatik und mentale Prozesse, 171-195. Tübingen, Stauffenburg.

— (1995) : «Interaction et syntaxe : Structures de participation et structures syntaxiques dans la conversation à plusieurs participants », in $\mathrm{D}$. Véronique et R. Vion (éds), Modèles de l'interaction verbale, 331-343. Aix-en-Provence, Publications de l'Université.

- (2008) : « Sprecherwechsel », in I. Kolboom, T. Kotschi, E. Reichel (éds), Handbuch Französich : Sprache - Literatur, - Kultur - Gesellschaft, 356359. Berlin, Erich Schmidt.

Pelose, G. C. (1987) : "The Functions of Behavioral Synchrony and Speech Rhythm in Conversation ", Research on language and social interaction, 20/11, 171-220.

SACKS, H. (1995) : Lectures on conversation, Oxford, Blackwell.

SACKS, H., SCHEGLOFF, E. A., JEFFERSON, G. (1974) : « A simplest systematics for the organization of turn-taking for conversation », Language, 50, 696-735.

SCHMALE, G. (2008) : «Constructions inachevées et transfert du tour de parole », $C M L F, 817-834$.

SCHEGLOFF, E. A. (1991) : «Reflections on Talk and Social Structure », in D. Boden et D. H. Zimmerman (éds), Talk and Social Structure. Studies in Ethnomethodology and Conversation Analysis, 44-70. Berkeley/Los Angeles, University of California Press.

— (1979) : «The relevance of repair to syntax-for-conversation », in T. Givón (éd), Syntax and semantics, vol. 12 : Discourse and syntax, 261-288. New York, Academic Press. 


\section{Annexe}

\section{Conventions de transcription}

\begin{tabular}{|c|c|}
\hline$().(.).(\ldots)$ & pause inférieure à une seconde, selon la durée \\
\hline (2.5) & pause mesurée \\
\hline $\begin{array}{l}\text { pay[er } \\
\text { [il y a }\end{array}$ & chevauchement \\
\hline là=mếme & enchaînement rapide (latching) \\
\hline 八 & intonation montante/descendante \\
\hline :.....:. & allongement d'un son, selon la durée \\
\hline VEUX & segment accentué \\
\hline l'ess- & auto-rupture \\
\hline$\langle(($ vite $)) x x>$ & commentaire valable pour $\mathrm{xx}$ \\
\hline $\begin{array}{l}\& \\
(x x x)\end{array}$ & $\begin{array}{l}\text { continuation du tour de parole du même locuteur à la ligne suivante } \\
\text { passage incompréhensible }\end{array}$ \\
\hline (y a enfin?) & transcription incertaine \\
\hline se lève* & description multimodale \\
\hline
\end{tabular}

